\title{
Mechanism of DNA damage responses induced by exposure to an oligonucleotide homologous to the telomere overhang in melanoma.
}

\author{
Ryan T Pitman ${ }^{1, *}$ Luke Wojdyla ${ }^{1}$, Neelu Puri ${ }^{1, *}$ \\ ${ }^{1}$ Department of Biomedical Sciences, University of Illinois College of Medicine, Rockford, Illinois \\ * These authors contributed equally to this study \\ Correspondence to: Neelu Puri, email: neelupur@uic.edu
}

Keywords: T-oligo, Tankyrase, TRF1, telomere, p53, melanoma

Received: May 21, $2013 \quad$ Accepted: June 1, $2013 \quad$ Published: June 3, 2013

This is an open-access article distributed under the terms of the Creative Commons Attribution License, which permits unrestricted use, distribution, and reproduction in any medium, provided the original author and source are credited.

\section{ABSTRACT:}

T-oligo, an 11-base oligonucleotide homologous to the 3'-telomeric overhang, is a novel, potent therapeutic modality in melanoma and multiple other tumor types. T-oligo is proposed to function in a manner similar to experimental disruption of the telomere overhang and induces DNA damage responses including apoptosis, differentiation and senescence. However, important components involved in T-oligo induced responses are not defined, particularly the role of p53, TRF1 and TRF2 in mediating the T-oligo induced responses. In MU, PM-WK, and MM-MC melanoma cells, exposure to T-oligo upregulates p53 expression and phosphorylation, resulting in cellular differentiation and activation of a caspase-mediated apoptotic cascade. However, siRNA-mediated knockdown of p53 completely blocks T-oligo induced differentiation and significantly decreases apoptosis, suggesting that p53 is an important mediator of T-oligo induced responses. In addition, we characterized the roles of telomere binding proteins, TRF1, TRF2, and tankyrase-1, in T-oligo induced damage responses. We demonstrate that tankyrase-1 activity is required for initiation of T-oligo induced damage responses including p53 phosphorylation and reduction of cellular proliferation. These results highlight TRF1, TRF2, tankyrase-1 and p53 as important elements in T-oligo mediated responses and suggest new avenues for research into T-oligo's mechanism of action.

\section{INTRODUCTION}

Telomeres and telomerase are areas of active research in tumor biology. Telomeres are structures that serve protective roles by allowing cells to distinguish chromosome ends from damaged DNA [1]. Disruption of the telomere results in multiple adverse consequences including potent DNA damage responses, chromosome fusion via non-homologous end joining, and genome instability [1]. Therefore, targeted disruption of the telomere or telomerase is an attractive therapeutic option [2]. Consequently, administration of T-oligo, an 11-base oligonucleotide homologous to the $3^{\prime}$ telomeric overhang, has been proposed as both a cancer therapeutic [3], and a method to study DNA damage responses induced by disruption of the telomere $[4,5]$.
Telomeres are composed of tandem nucleotide repeats (TTAGGG) at the end of chromosomes [1] that form a protective T-loop at the single-stranded $3^{\prime}$ end. The T-loop caps and protects the telomere end through recruitment of the shelterin complex, composed of telomeric repeat binding factors 1 and 2 (TRF1 and TRF2), POT1, TIN1, TPP1 and RAP1 [6]. TRF1 and TRF2 directly bind the duplex DNA region of T-loops and function as negative regulators of telomere length by preserving loop structure integrity and preventing telomerase access to the telomere [7-9]. Expression of dominant negative TRF2 induces uncapping of the telomere overhang and initiates DNA damage responses, possibly by disruption of the T-loop and exposure of the telomere overhang, a process mediated in part through ATM and its effector protein p53 [5, 10]. Conversely, overexpression of TRF2 in telomerase negative cells 
leads to an increased rate of telomere shortening and less efficient repair of single-stranded breaks (SSBs) in telomeric DNA [11]. TRF1 functions in a similar manner by binding telomeric DNA and stabilizing the shelterin complex [6]. Both TRF1 and TRF2 are thought to be key mediators of T-loop integrity and their disruption could lead to downstream DNA damage responses.

Tankyrase-1, a telomere-specific poly(ADPribosyl) polymerase (PARP), acts as an inhibitor of TRF1 by catalyzing the addition of poly (ADP-ribose) (PAR) groups to TRF1 and preventing it from binding telomeric DNA [12]. Overexpression of tankyrase-1 leads to telomerase binding and telomere elongation in telomerase positive cells [13], presumably as a consequence of TRF1 inhibition $[14,15]$. Consistent with this data, inhibition of tankyrase-1 induces telomere shortening in the presence of telomerase, and thus its inhibition has emerged as a prospective cancer therapy [16]. Tankyrase-1 inhibitors, XAV939 and 3-aminobenzamide (3AB), block telomerase from accessing telomeric DNA [13], presumably by preventing PARsylation of TRF1 [12] and cause a downstream DNA damage response $[17,18]$.

T-oligo has been proposed as a means for studying downstream DNA damage responses caused by exposure of the telomere overhang. T-oligo induces potent DNA damage responses $[4,19]$ including transient cell cycle arrest, adaptive differentiation, replicative senescence and apoptosis [3, 4, 20, 21]. T-oligo accumulates in the nucleus and initiates downstream signaling through p95/ Nbs1, p16, pRb, p53, p73 and p21 [3, 4, 20-22]. However, induction of DNA damage responses by T-oligo does not require damaged telomeric DNA [19], and T-oligo may lead to an increase in mean telomere length [4]. Further, T-oligo has been proposed as a cancer therapeutic because it induces apoptosis and differentiation and specifically targets malignant cells $[3,5,23]$.

At present, it is unclear how introduction of T-oligo into the cell results in a downstream DNA damage response. T-oligo may function as a "replacement" signal in place of telomeric DNA obscured by overexpressed telomerase [5]. We propose that T-oligo initiates a DNA damage response through recruitment of telomeric structures away from the telomere with subsequent exposure of the telomere overhang. To investigate the connection between telomere exposure and T-oligoinduced DNA damage responses, we utilized tankyrase-1 inhibitors, XAV939 and 3AB, to prevent tankyrase-1 mediated dissociation of TRF1 from telomeric DNA. This would, in effect, prevent dissociation of the shelterin complex and disruption of the telomere overhang which, under normal circumstances, induces a DNA damage response [6]. We then exposed cells to T-oligo to determine if tankyrase-1-mediated dissociation of TRF1 from the telomere is required for T-oligo to elicit a DNA damage response.

Further, the exact signaling pathways through which
DNA damage responses, mediated by T-oligo, proceed are still undefined. p53, a DNA damage response mediator, has been proposed to play an important role in T-oligo induced DNA damage responses as it is upregulated and phosphorylated in response to T-oligo [5]. However the role of p53 in T-oligo-mediated anticancer responses in melanoma has not been demonstrated. Thus, we investigated the downstream effects of T-oligo, including differentiation and the apoptotic caspase cascade, before and after siRNA mediated p53 knockdown, in p53 expressing MU, PM-WK, and MM-MC melanoma cells.

In this study, we improve our understanding of the DNA damage pathways induced by T-oligo through an examination of the role of p53 in apoptosis and differentiation in melanoma cells. Further, we examine the role of the telomere binding proteins, TRF1 and TRF2, and also delineate the requirement of tankyrase- 1 activity for T-oligo's downstream effects.

\section{RESULTS}

\section{Treatment with T-oligo induces p53 expression and phosphorylation in melanoma cells.}

Exposure of the telomere overhang induces a strong DNA damage response in malignant cells including apoptosis and differentiation [3-5]. T-oligo induced DNA damage responses are believed to progress through ATM, p53, E2F1 and p95/NBS1 [3, 5, 19, 24], however, the roles of ATM and p53 are unclear $[4,22]$. To define the role of p53 in melanoma cells, we exposed p53-expressing MU, MM-MC and PM-WK melanoma cells to T-oligo for various time points and then performed immunoblotting. In MU cells, T-oligo increases E2F1, a driver of p53 expression $[25,26]$, after $24 \mathrm{hrs}$ of exposure in addition to upregulation of p53 expression and phosphorylation after 24 and 48 hrs of exposure (Fig 1A). T-oligo also upregulates p21, a transcriptional target of p53 [27], after 24 and $48 \mathrm{hrs}$ of exposure in MU melanoma cells (Fig 1A). PM-WK cells exhibit a similar response with increased E2F1 after 24 hrs of exposure to T-oligo and upregulation of p53 expression and its phosphorylation at $48 \mathrm{hrs}$ of exposure (Fig 1B). A similar response is demonstrated in MM-MC cells with significant upregulation of E2F1 after $24 \mathrm{hrs}$ and p53 phosphorylation after $48 \mathrm{hrs}$ of exposure to T-oligo (Fig 1C). These results suggest an integral role of p53 in T-oligo induced DNA damage responses.

\section{Exposure to T-oligo induces a caspase cascade}

Studies in our laboratory and others show that T-oligo selectively induces apoptosis in multiple types of malignant cells including melanoma, prostate, NSCLC, ovarian and breast [3-5, 22, 23, 28-30]. However, few 
insights are available into the involvement of an apoptotic cascade in T-oligo induced apoptosis in melanoma. To further define and understand the caspases induced by T-oligo in p53-expressing melanoma cells, we analyzed MU and PM-WK melanoma cells for induction of a caspase cascade. In PM-WK cells, survivin, an inhibitor of apoptosis that is overexpressed in melanoma and blocks activation of caspase-9 [31, 32], is downregulated after $72 \mathrm{hrs}$ of exposure to T-oligo (Fig 2A), with subsequent decrease of the caspase-9, caspase-3, and caspase-7 proenzymes after 96 hrs of treatment with T-oligo (Fig 2B). In addition, MU cells also exhibit a decrease in caspase-3 and caspase-7 proenzymes after $96 \mathrm{hrs}$ of exposure to T-oligo (Fig 2C). To validate and quantify caspase-3 activation and activity, we measured caspase-3 catalytic activity using the colorimetric CaspACE assay. In MU cells, increased caspase-3 activity is observed after both 72 and $96 \mathrm{hrs}$ of treatment with T-oligo (Fig 2D). These results establish involvement of the caspase cascade in T-oligo induced apoptosis in melanoma.

\section{Induction of melanoma differentiation after exposure to T-oligo.}

Although much attention is given to T-oligo induced apoptosis, the development of metastatic melanoma is often coupled with a loss of melanocyte differentiationspecific antigens [33] that are utilized in immunotherapymediated treatment of melanoma [34]. Previous studies identify an important link between exposure of the telomere overhang with differentiation in melanoma [35-37], and we suggest that T-oligo could also mediate differentiation of melanoma cells [3]. Additionally, differentiation of melanoma cells is associated with slowed growth and reduced tumorigenicity $[36,37]$ and is the target of melanoma vaccine therapy [34].

Currently, it is unclear if treatment with T-oligo can cause uniform differentiation across all melanoma cell types. To determine the extent of T-oligo induced differentiation, MU, PM-WK and MM-MC melanoma cells were exposed to T-oligo and subsequently
A

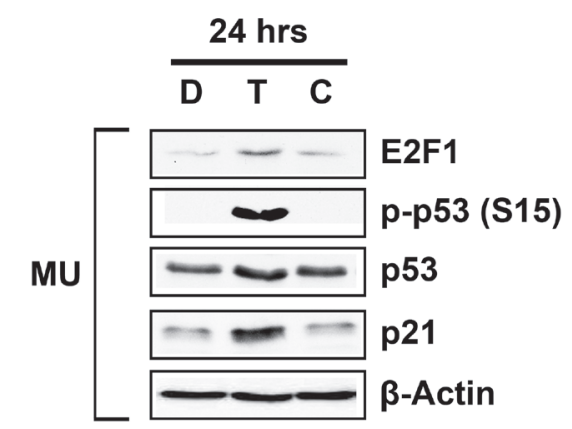

B

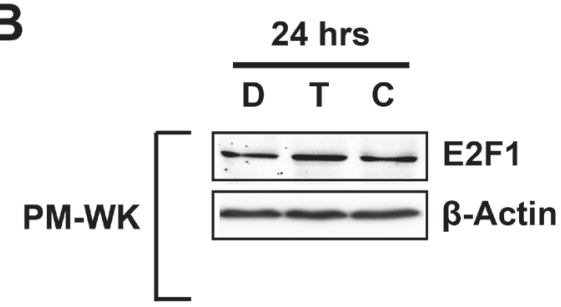

C

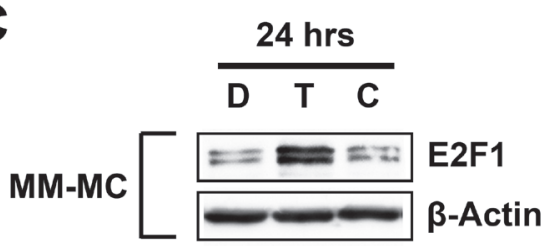

48 hrs
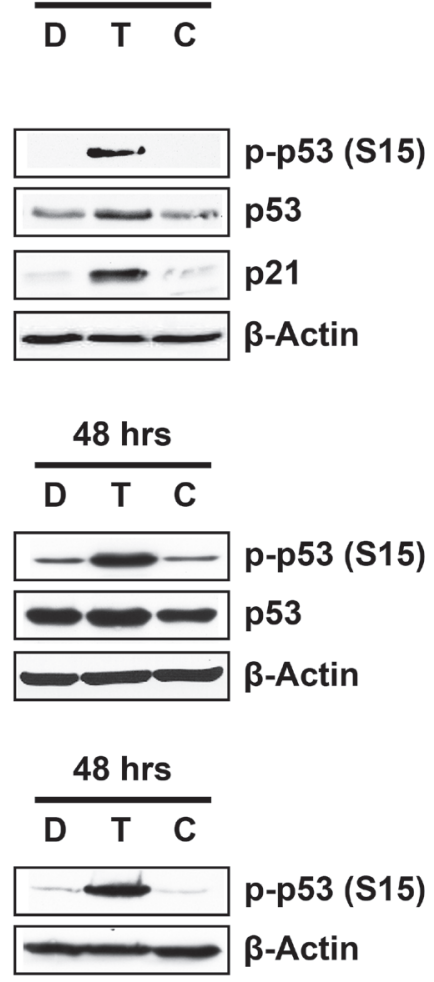

Figure 1: Induction of E2F1, p53, p21 and phosphorylation of p53 (Ser15) in melanoma cells. MU, MM-MC or PM-WK melanoma cells were treated with $40 \mu \mathrm{M}$ of T-oligo (T), complementary oligo (C) or an equal volume of diluent (D) for 24-48 hrs and subsequently prepared for immunoblotting. (A) MU cells demonstrate upregulation of p53 at 24 hrs (1.5-fold) and 48 hrs (2-fold) with corresponding increase in phosphorylation of p53 (ser15) of 18-fold and 25-fold at 24 and 48 hrs, respectively. Further, p21, a downstream transcription product of p53, is increased 2.5-fold and 5-fold in MU cells at 24 and 48hrs, respectively. E2F1 expression is also increased by 2.5 -fold at $24 \mathrm{hrs}$. (B) T-oligo induces a similar increase in p53 and phospho-p53 (ser15) by 1.5 -fold and 4.8 -fold respectively at 48 hrs in PM-WK cells, with an increase in E2F1 expression by 1.5-fold at $24 \mathrm{hrs}$ in PM-WK cells. (C) MM-MC also exhibit upregulation of E2F1 (3.5-fold) after 24hrs of exposure to T-oligo along with a significant 17-fold increase in p53 phosphorylation after 48 hrs of exposure to T-oligo. Experiments were performed in triplicate with representative results displayed. 
immunoblotted for expression of the melanoma differentiation proteins MART-1, TRP-1, TRP-2, and tyrosinase. $\mathrm{MU}$ melanoma cells exposed to T-oligo express MART-1 after 24 hrs (Fig 3A) with additional expression of TRP-1 (Fig 3B), tyrosinase and TRP-2 after 96 hrs (Fig 3C). Interestingly, PM-WK cells express only tyrosinase after $48 \mathrm{hrs}$ of exposure to T-oligo (Fig 3D), while tyrosinase and TRP-2 are increased in MM-MC cells over the entire course of $72 \mathrm{hrs}$ of exposure to T-oligo (Fig 3E). These results provide compelling evidence for strong and progressive T-oligo-induced differentiation in p53-expressing melanoma.

\section{Loss of T-oligo induced differentiation and apoptosis after p53 knockdown.}

While p53 is proposed to be a primary mediator of T-oligo-induced DNA damage responses in breast cancer [5], the role of p53 in melanoma is unclear as p53-deficient MM-AN cells undergo apoptosis after upregulation of p73 [4]. To demonstrate a definitive role for p53 in melanoma differentiation and apoptosis in response to T-oligo exposure, MU cells were transfected with either anti-p53 siRNA or control siRNA for 12 hrs. Knockdown of p53 expression was confirmed by a 10- and 2.2-fold downregulation at 72 and 96 hrs after transfection, respectively (Fig 4A). MU cells treated with siRNA against cells were then exposed to T-oligo for 72 hrs and subsequently stained with propidium iodide and analyzed by FACS analysis for apoptosis. Loss of p53 decreased T-oligo induced apoptosis by 45\% (Fig 4C), indicating that it has a substantial, although perhaps not exclusive, role in apoptosis. Additionally, p53 knockdown eliminated the T-oligo-induced expression of tyrosinase and TRP-1 (Fig 4B). This data suggests that p53 plays a vital role in T-oligo-induced apoptosis and differentiation in melanoma.

\section{Inhibition of tankyrase-1 blocks T-oligo-induced DNA damage responses.}

Although the T-oligo induced DNA damage responses are well established [3-5, 19-21, 29], the initiating events that lead to T-oligo induced DNA damage responses are not defined. T-oligo is proposed to function in a manner similar to exposure of the telomere overhang [5] as T-oligo induced DNA damage responses are similar to the effects of TRF2 disruption [5, 19]. TRF1 functions in similar manner as TRF2 by mediating the association of the shelterin complex with the telomere [6] and is PARsylated by tankyrase-1 which leads to its dissociation from the telomere [12], exposure of the telomere
A

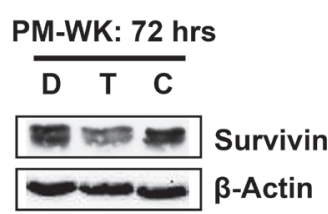

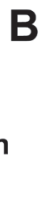
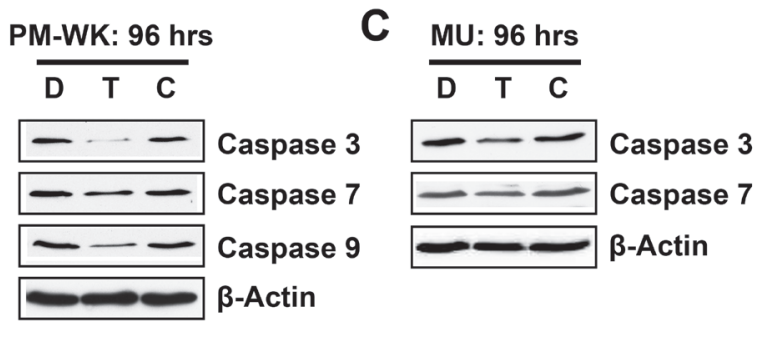

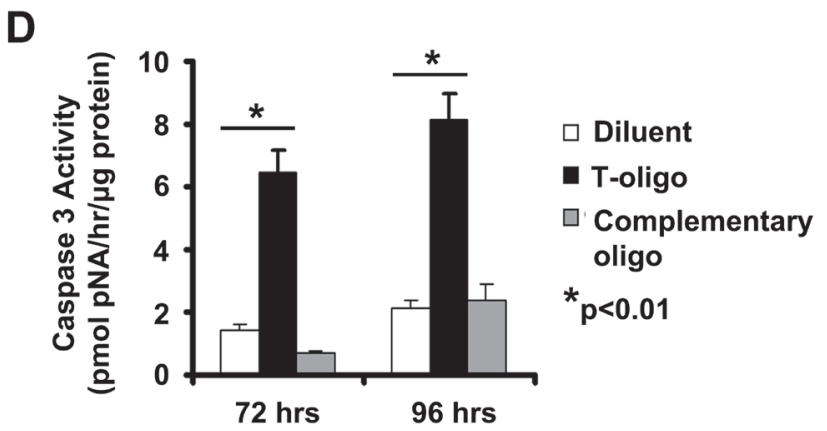

Figure 2: T-oligo initiates a caspase cascade in melanoma cells. Melanoma cells were treated with of T-oligo (T), complimentary oligo (C) or an equal volume of diluent (D) for indicated times and cell lysates were immunoblotted for detection of survivin and caspase proenzymes. (A) T-oligo treatment in PM-WK caused downregulation in survivin (1.5-fold) (B) followed by a 9.3, 2.9, and 1.5-fold decrease in caspase-3, 7, and 9 proenzymes respectively, at $96 \mathrm{hrs}$ of incubation with T-oligo as compared to the complimentary oligonucleotide. (C) Similarly, MU melanoma cells also demonstrate a decrease in caspase-3 (3.3-fold) and 7 (2.6-fold) at 96 hrs of treatment with T-oligo as compared to the complementary oligo. (D) To confirm increased caspase-3 catalytic activity in response to T-oligo treatment, MU cells were treated as described above for 72 or $96 \mathrm{hrs}$ and subsequently collected and analyzed for caspase-3 activity using the CaspACE assay system. Caspase- 3 activity is increased by 4.5 -fold and 4 -fold at 72 and $96 \mathrm{hrs}$, respectively. Immunoblots are representative of at least three independent experiments. Analysis of caspase- 3 activity was done in triplicate with error bars representing standard deviation. $(*=p<0.01)$ 
overhang and recruitment of telomerase [13]. Inhibitors of tankyrase-1 prevent PARsylation of TRF1 [12] thereby preventing exposure of the telomere overhang with consequences including an inability of telomerase to bind the telomere and subsequent development of senescence $[13,38]$. To determine the role of telomere exposure in induction of T-oligo's DNA damage responses, we utilized XAV939 and 3AB, inhibitors of tankyrase-1.

MU melanoma cells cultured as described above and treated with XAV939 or 3-aminobenzamide $(3 \mathrm{AB})$ demonstrate reduced cellular proliferation (Fig 5A,B) [18]. However, simultaneous treatment with XAV939 or 3AB completely blocked T-oligo-induced inhibition of cellular proliferation since cells treated with T-oligo and 3AB or XAV939 are not significantly different from cells treated with diluent or complementary oligonucleotide and 3AB or XAV939 as determined by one-way ANOVA. Further, treatment with XAV939 or $3 \mathrm{AB}$ prevented T-oligo induced p53 phosphorylation (Fig 5C). These results suggest that tankyrase-1 must be able to PARsylate and remove TRF1 from the telomere in order for T-oligo to induce a DNA damage response. Immunoblotting of MU cells treated with T-oligo demonstrate upregulation of TRF2 (Fig $5 \mathrm{D})$, and treatment with XAV939 or $3 \mathrm{AB}$ blocked this effect. This is the first demonstration of T-oligo mediated upregulation of TRF2. These results are novel and suggest an important requirement of tankyrase-1 activity leading to TRF1 dissociation from the telomere for induction of T-oligo-mediated DNA damage responses.

\section{DISCUSSION}

Investigation into telomere biology is an important area for understanding tumor biology and identification of novel therapeutic modalities [2]. T-oligo has important therapeutic potential in multiple tumor types, but its mechanism is not fully defined. In this manuscript, we demonstrate induction of a caspase cascade and upregulation of differentiation markers in p53-expressing MU, PM-WK, and MM-MC cells. Further, we verify the importance of p53 in the downstream DNA damage responses induced by T-oligo. We also inhibit tankyrase-1 activity and demonstrate a requirement for tankyrase-1 activity in T-oligo induced DNA damage responses.

p53 expression and phosphorylation are hallmarks of the DNA damage response seen in cells exposed to DNA alkylating agents [39], exposure of the telomere overhang through disruption of the T-loop structure [40], and exposure to T-oligo [4, 20]. However, its role

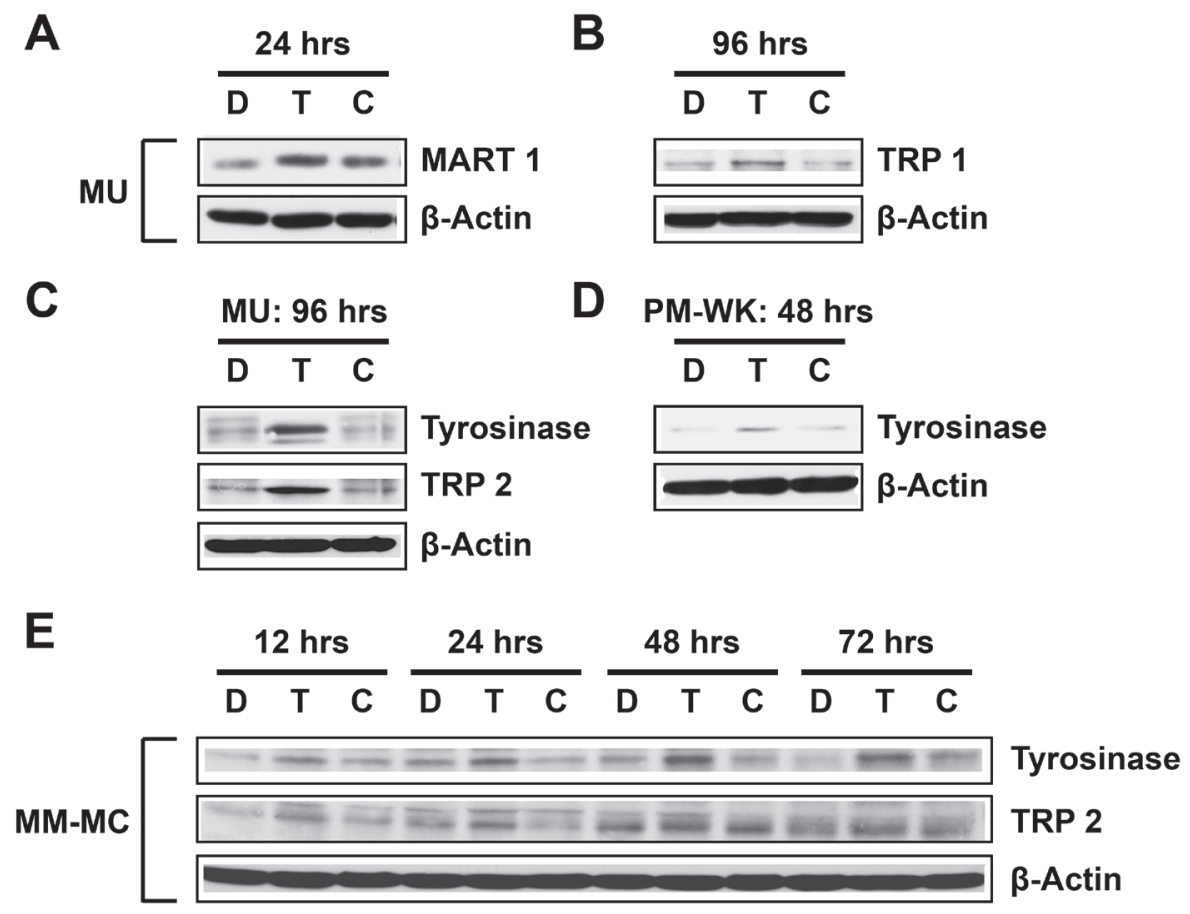

Figure 3: T-oligo strongly induces differentiation in multiple melanoma cell lines. Melanoma cells were exposed to 40 $\mu \mathrm{M}$ of T-oligo (T), complementary oligo (C) or an equal volume of diluent (D) for the indicated times and samples were collected for immunoblotting. (A) In MU melanoma cells, T-oligo upregulates MART-1 by 2.7-fold at $24 \mathrm{hrs,} \mathrm{(B)} \mathrm{and} \mathrm{TRP-1} \mathrm{by} 2.5$-fold at 96 hrs. (C) Additional upregulation of TRP-2 (2.9-fold) and tyrosinase (2.3-fold) is observed in MU cells after 96 hrs of treatment with T-oligo. (D) PM-WK cells also demonstrate 3 -fold increase in tyrosinase after only $48 \mathrm{hrs}$ of exposure to T-oligo. (E) To better understand the time course of T-oligo-induced differentiation, MM-MC melanoma cells were immunoblotted for the expression of TRP-1 and TRP-2 over the course of $72 \mathrm{hrs}$ of exposure to T-oligo. Both TRP-1 and TRP-2 are increased over the entire course of 12 to $72 \mathrm{hrs}$ of T-oligo treatment (2.9-fold and 2.6-fold, respectively). Immunoblots are representative of at least three independent experiments. 
in T-oligo induced apoptosis has been questioned as lack of p53 does not block T-oligo induced apoptosis in cells that do not express p53 $[3,4,19,22]$. In cells that do express p53, we suggest that p53 is still likely to play an important role in driving DNA damage responses. In this study, p53-expressing melanoma cells undergo a caspase cascade and exhibit signs of differentiation upon exposure to T-oligo. Further, knockdown of p53 completely blocks T-oligo induced differentiation and significantly decreases apoptosis after exposure to T-oligo. However, the inability for p53 knockdown to completely block T-oligo induced apoptosis suggests other pathways likely contribute. p73, a p53 homologue, is upregulated in p53-deficient melanoma cells after exposure to T-oligo and transfection of a dominant-negative p73 significantly decreases T-oligo induced apoptosis [4]. Alternatively, the WRN helicase, which responds to disruption in the telomere overhang, is also suggested to play a role in T-oligo induced apoptosis [24], although the mechanism whereby WRN signals for a DNA damage response is unclear. Although these results suggest that p53 is likely to play an important role in T-oligo induced DNA damage responses, further research is required to understand other key proteins involved in T-oligo induced DNA damage responses.
Despite an increasing understanding of T-oligo's DNA damage responses [4, 19-21] and demonstration of T-oligo's potential as a therapeutic in multiple tumor types [3, 5, 22, 28, 29], little is understood of how T-oligo activates the DNA damage responses. ATM and WRN, a helicase protein, have been implicated $[19,24]$ as primary drivers, but it is unclear how T-oligo is able to signal for DNA damage. Others have suggested that T-oligo mimics an exposed 3' telomere overhang, thus initiating the DNA damage pathway $[19,41]$. This conclusion is based on observations that the DNA damage responses induced by T-oligo and disruption of the telomere overhang by transfection with a dominant-negative TRF2 follow similar pathways and time-courses [5, 19]. However, administration of T-oligo does not cause degradation of the telomere overhang, seen in TRF2 disruption [19]. Further, these observations do not account for an increase in mean telomere length [4] or transient telomerase activation in response to T-oligo administration [42]. In light of these observations, we suggest that T-oligo does not simply "mimic" the telomere overhang.

TRF1 and TRF2 function to stabilize the telomere overhang through their association with the shelterin complex [6] and tankyrase-1 is proposed to allow
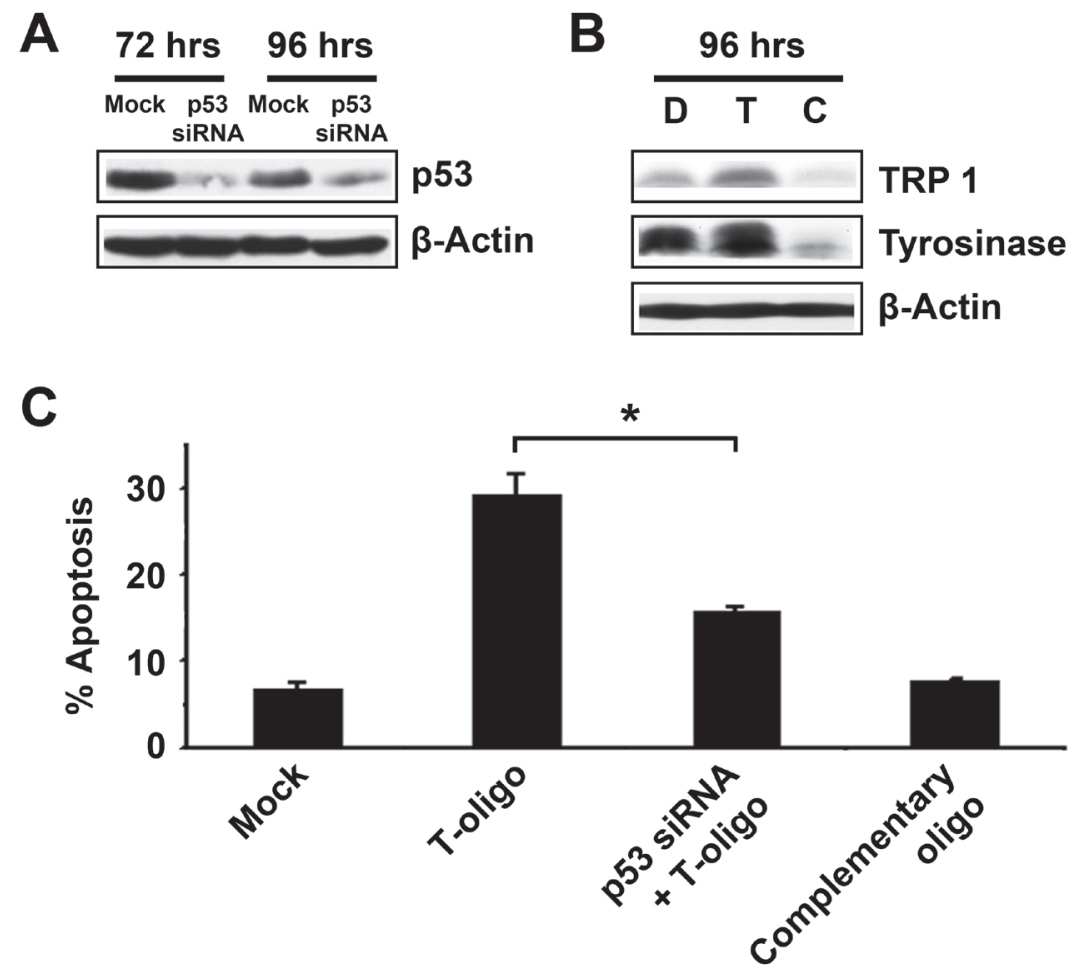

Figure 4: T-oligo induced differentiation and apoptosis in melanoma is dependent upon the presence of p53. MU melanoma cells were transfected with p53 specific siRNA or mock siRNA and analyzed using immunoblotting. (A) Down regulation of p53 expression can be seen at $72 \mathrm{hrs}$ (10-fold) and $96 \mathrm{hrs}$ (2.2-fold). (B) MU cells treated with siRNA against p53 for 12 hrs were then exposed to T-oligo for $96 \mathrm{hrs}$ and immunoblotted for TRP-1 and Tyrosinase. p53 knockdown completely blocks T-oligo-induced differentiation. (C) p53-deficient MU cells were then treated with T-oligo for $72 \mathrm{hrs,} \mathrm{collected} \mathrm{and} \mathrm{stained} \mathrm{with} \mathrm{propidium} \mathrm{iodide} \mathrm{for} \mathrm{FACS} \mathrm{analysis.}$ Knockdown of 553 decreased T-oligo-induced apoptosis by $45 \%$. Immunoblots are representative of at least three independent experiments. Determination of apoptosis was performed in quadruplicate with error bars representing standard deviation. Statistical analysis by the Student's T-test found a significant difference between cells treated with T-oligo and those treated with both siRNA against p53 and T-oligo. $(*=\mathrm{p}<0.001)$ 
telomerase to function through destabilization of shelterin through PARsylation of TRF1 [13, 14]. Tankyrase-1 inhibitors prevent TRF1 dissociation from the telomere [43] and are thought to contribute to apoptosis through increased sister chromatid exchanges at the telomeres [17], or inhibition of telomerase [43]. In this study, we confirm a decrease in cellular proliferation by tankyrase-1 inhibition, but also demonstrate that inhibition of tankyrase-1 abrogates T-oligo-induced p53 phosphorylation and decreased cellular proliferation. These results suggest that tankyrase-1 function is vital to T-oligo mediated anticancer effects.

At present the role of tankyrase-1 in T-oligo mediated DNA damage responses is unclear. We suggest that T-oligo binds to shelterin associated proteins following PARsylation of TRF1, and recruits the shelterin complex away from the telomere (Fig 6). In this uncapped state, telomeres are exposed, thus providing an opportunity for recognition as DNA damage foci by ATM or WRN [19, 24]. Alternatively, this allows for binding of the telomere by telomerase, previously suggested by evidence that T-oligo increases mean telomere length [19], possibly through a transient increase in telomerase activity [42]. An increase in mean telomere length may also explain our finding of increased TRF2 expression after exposure to T-oligo since elongated telomeres require increased TRF2 for stability [6]. This is in contrast to treatment with TRF2DN which results in telomere degradation [4], or other studies that show upregulation of TRF2 maintains the telomere duplex structure. In the present experiment, we propose that inhibition of tankyrase-1 maintains the integrity of the telomere, thus completely blocking T-oligo induced DNA damage responses and suggesting that destabilization of the telomere overhang is a requirement for T-oligo to act as a therapeutic agent. These results suggest that T-oligo functions to prevent restabilization following dissociation of TRF1 from the shelterin complex, leading to disruption of the T-loop and downstream DNA damage responses. We therefore suggest additional experiments involving disruption of TRF1 to further define its role in T-oligo-induced DNA damage responses.

In addition to PARsylating TRF1, tankyrase-1 contributes many functions to cellular physiology including PARsylation of axin, targeting it for ubiquitination and degradation, and inhibiting the Wnt pathway causing growth inhibition [18] and regulation of mitosis [44]. At present, there is no data to suggest that

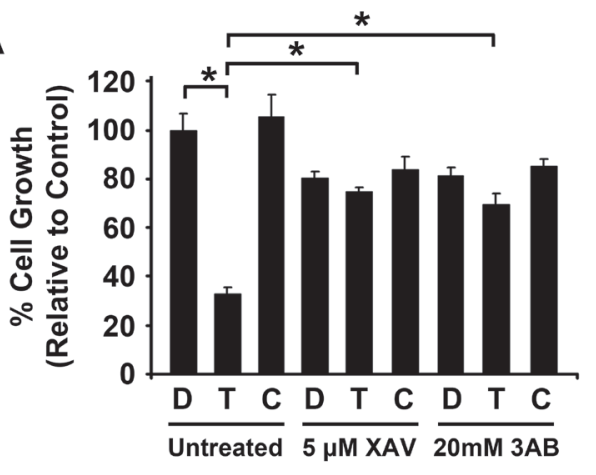

B

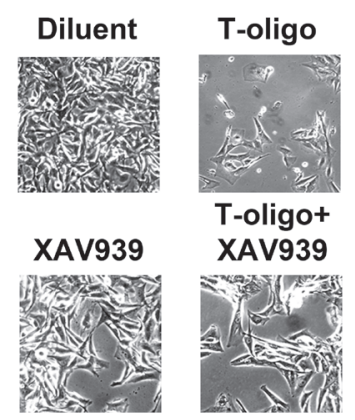

C
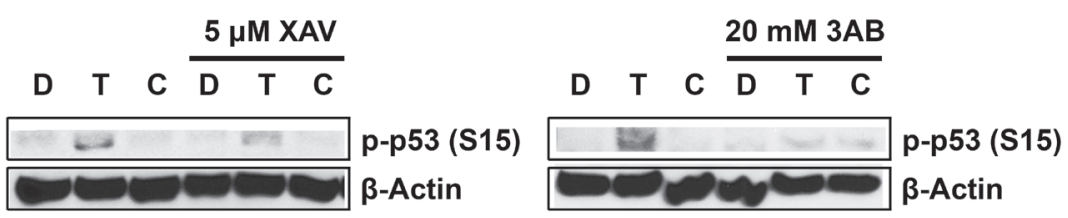

D

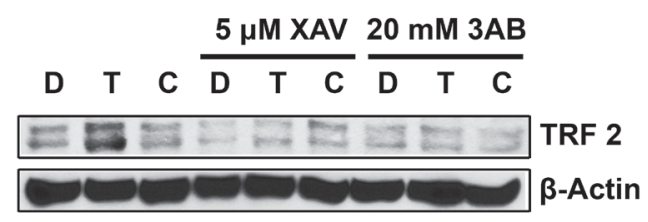

Figure 5: Inhibition of Tankyrase-1 prevents T-oligo induced DNA damage responses. MU cells were treated with $5 \mu \mathrm{M}$ XAV939 or $20 \mathrm{mM} 3 \mathrm{AB}$ prior to treatment with $40 \mu \mathrm{M}$ T-oligo or complementary oligo. (A) Treatment with both XAV939 and 3AB decreased cellular proliferation in MU cells. However, treatment of MU cells with XAV939 or 3AB with T-oligo completely blocks T-oligoinduced decrease in cellular proliferation. (B) Microphotographs depicting effect of XAV939 treatment on T-oligo-induced reduction in cellular proliferation. (C) Treatment with XAV939 or 3AB also blocks T-oligo-induced phosphorylation of p53 (ser15). (D) Exposure of MU cells to T-oligo induces a 2.6-fold increase in TRF2 levels, but this effect is also blocked by treatment with XAV939 or 3AB. The effects of T-oligo after XAV939 or $3 \mathrm{AB}$ treatment are not significant by analysis using one-way ANOVA. Immunoblots are representative of at least three independent experiments. $(*=p<0.001)$ 
T-oligo could function to modulate these other pathways; however these findings suggest new avenues of research.

In this study, we document considerable advances in understanding the mechanism of T-oligo induced DNA damage responses. p53 is shown to be an important contributor to T-oligo induced apoptosis and differentiation in p53-expressing melanoma cells. Further, inhibition of tankyrase-1 prevents T-oligo induced DNA damage responses, suggesting a new paradigm in understanding T-oligo's mechanism of action. These results are novel, important and provide new insight into telomere biology and the mechanism whereby T-oligo induces DNA damage responses.

\section{MATERIAL AND METHODS:}

\section{Oligonucleotides:}

11-base DNA oligonucleotides homologous (pGTTAGGGTTAG) (T-oligo) and complementary (pCTAACCCTAAC) (complementary oligo) to the 3' telomere overhang sequence were obtained from Midland Certified Reagent Company (Midland, TX) for cell culture experiments.
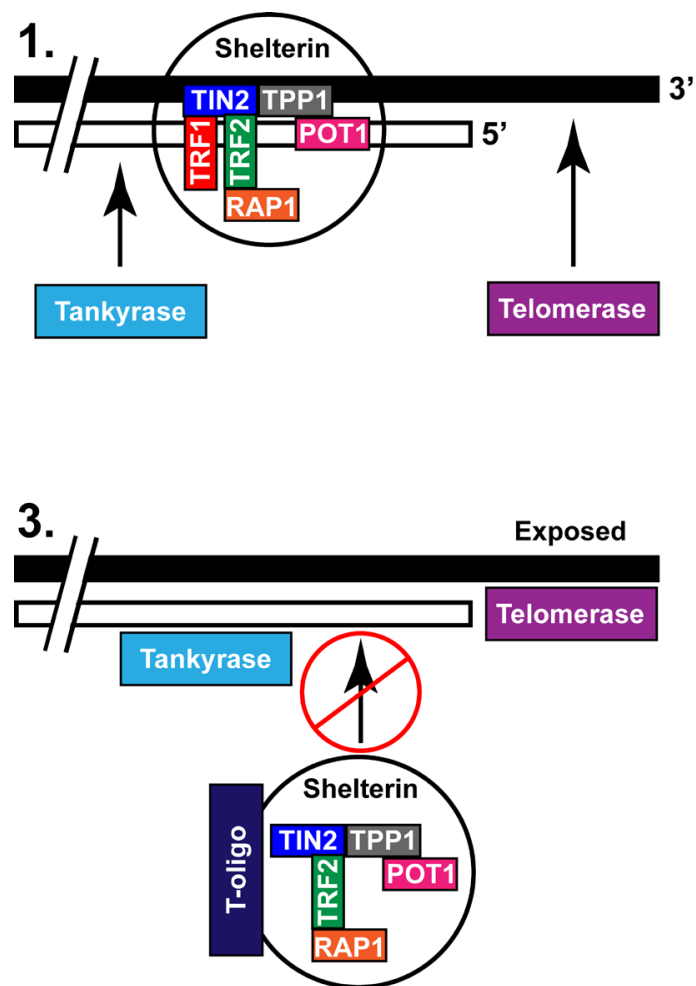

\section{Cell culture:}

MU, PM-WK, and MM-MC melanoma cells were obtained by explant culture [45] and grown in MEM (\#32561-037) with 10\% FBS (v/v) (\#16000-044) from Gibco (Gaithersburg, MD, USA) and 1\% antibiotic/ antimycotic (v/v) (\#15240) obtained from Invitrogen (Grand Island, NY) as described previously [3].

\section{Immunoblotting and antibodies:}

Melanoma cells were grown in MEM media containing 5\% FBS and treated with diluent (water), 40 $\mu \mathrm{M}$ complementary oligonucleotide, or $40 \mu \mathrm{M}$ T-oligo for various time points. After which, cells were harvested and protein lysates immunoblotted as described previously [46]. p53 (pantropic) antibody (clone DO-1, \#OP43A) was obtained from EMD Millipore (Billerica, MA). phospho-p53 (Ser 15) (\#:9284) polyclonal antibodies, cleaved Caspase-3 (Clone 5A1E, Rabbit mAb \#9664), cleaved Caspase-7 (Clone D6H1, Rabbit mAb \#8438), and cleaved Caspase-9 (Clone D2D4, Rabbit mAb \#7237) were purchased from Cell Signaling Technology (Danvers, MA). For detection of differentiation, the following antibodies were used; tyrosinase monoclonal antibody (clone T311: Novacastra $^{\mathrm{TM}}$, Leica Biosystems Newcastle
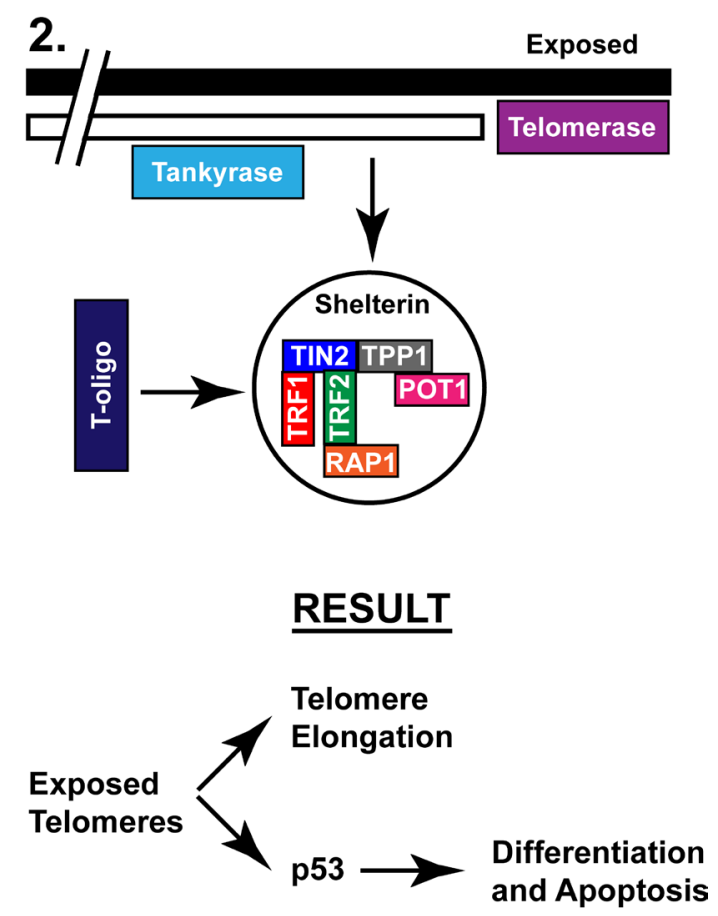

Figure 6: Proposed mechanism of T-oligo induced activation of the DNA damage response through recruitment of the shelterin complex. The shelterin complex, composed of TRF1, TRF2, TIN1, POT1, TPP1, and RAP1 stabilizes the telomere and prevents telomere overhang exposure. Tankyrase causes dissociation of shelterin through PARsylation of TRF1, leading to telomere overhang disruption. T-oligo is then able to bind the shelterin complex and maintain exposure of the telomere overhang which leads to either telomere elongation or DNA damage responses including apoptosis or differentiation. 
upon Tyne, UK), TRP-1 (sc 10443) and TRP-2 (sc 10452) polyclonal antibodies (Santa Cruz Biotechnology, Santa Cruz, CA), and MART-1/Melan-A monoclonal antibody (clone M2-7C10: Signet, Dedham, MA). The TRF1 antibody was a generous gift from Dr. Jan Karlseder, and TRF2 antibody was purchased from (\#NB110-57130: Novus Biologicals, Littleton, CO). Beta-actin antibody (\#A-5441), Sheep anti-Mouse IgG (\#NA931V), and Donkey anti-Rabbit IgG (\#NA934V) were purchased from GE healthcare (Piscataway, NJ), and all other chemicals were obtained from Sigma Aldrich (St. Louis, MO). Densitometry was performed using ImageJ software (National Institutes of Health, Bethesda, Maryland, USA).

\section{Transfection of p53 siRNA:}

MU melanoma cells were transfected with four pooled siRNA duplexes, from Dharmacon (Lafayette, CO) against p53 (\#M-003329-03-0005) in optiMEM (\#11058021, Invitrogen, Carlsbad, CA) using oligofectamine (\#12252-011: Invitrogen, Carlsbad, CA). Mock transfection was done in parallel using signal silence control siRNA (\#6568S: Cell Signaling Technology, Beverly, MA).

\section{Determination of Apoptosis:}

Melanoma cells were cultured as described previously and transfected with siRNA for $12 \mathrm{hrs}$ and subsequently treated with diluent (water), $40 \mu \mathrm{M}$ T-oligo, or $40 \mu \mathrm{M}$ complementary oligo for $72 \mathrm{hrs}$. Cells were then stained with propidium iodide (\#81845: Sigma-Aldrich; St. Louis, MO) and analyzed by a Becton Dickinson FACS Scan. Apoptosis was studied by determining sub $\mathrm{G}_{\mathrm{o}} / \mathrm{G}_{1}$ DNA content by FACS analysis as described previously [3]. Statistical significance was determined using the paired Student's T-test with significance established at alpha $=0.05$.

Apoptosis was also determined by evaluation of caspase-3 activity using the CaspACE assay system (\#G7351) from Promega (Madison, Wisconsin). Quadruplicate cultures of melanoma cells were treated with diluent (water), $40 \mu \mathrm{M}$ control oligonucleotide, or 40 $\mu \mathrm{M}$ T-oligo for 72 or $96 \mathrm{hrs}$. Cells were then collected by centrifugation, washed and lysed by repeated freeze/thaw cycles. The lysate was then clarified by centrifugation and the supernatant was used for protein estimation and for the caspase assay. This assay uses Ac-DEVD-pNA as a substrate and colorimetrically measures the release of free pNA. Statistical significance was determined using the paired Student's T-test with significance established at alpha $=0.05$.

\section{Tankyrase-1 inhibition:}

For measuring the effect of tankyrase-1 inhibitors on T-oligo induced cellular proliferation in MU melanoma cells, triplicate cell cultures were plated at $6 \times 10^{4}$ cells in $60 \mathrm{~mm}^{2}$ dishes in MEM with $10 \%$ FBS and treated with tankyrase-1 inhibitors, XAV939 $(5 \mu \mathrm{M})$ or $3 \mathrm{AB}$ $(20 \mathrm{mM})$, or equal amount of diluent (water), and a final concentration of $40 \mu \mathrm{M}$ T-oligo, complementary oligo, or equal amount of diluent (water). After $24 \mathrm{hrs,} \mathrm{cells}$ were prepared for immunoblotting. After $96 \mathrm{hrs,} \mathrm{cells}$ were trypsinized and counted using a hemocytometer in the presence of trypan blue. Statistical significance was determined using the one-way ANOVA with significance established at alpha $=0.05$.

\section{ACKNOWLEDGMENTS}

The authors declare no conflicts of interest. Research reported in this publication was supported by National Institute of Arthritis and Musculoskeletal and Skin Diseases of the National Institutes of Health under Award Number 7R03AR050110. The content is solely the responsibility of the authors and does not necessarily represent the official views of the National Institutes of Health. The authors would like to acknowledge the Research Open Access Publishing (ROAAP) Fund of the University of Illinois at Chicago for financial support towards the open access publishing fee for this article.

\section{REFERENCES}

1. de Lange T. Protection of mammalian telomeres. Oncogene. 2002; 21(4):532-40.

2. Ruden $M$ and Puri N. Novel anticancer therapeutics targeting telomerase. Cancer Treat Rev. 2013; 39(5):44456.

3. Puri N, Eller MS, Byers HR, Dykstra S, Kubera J and Gilchrest BA. Telomere-based DNA damage responses: a new approach to melanoma. FASEB J. 2004; 18(12):137381.

4. Eller MS, Puri N, Hadshiew IM, Venna SS and Gilchrest BA. Induction of apoptosis by telomere 3' overhangspecific DNA. Exp Cell Res. 2002; 276(2):185-93.

5. Yaar M, Eller MS, Panova I, Kubera J, Wee LH, Cowan KH and Gilchrest BA. Telomeric DNA induces apoptosis and senescence of human breast carcinoma cells. Breast Cancer Res. 2007; 9(1):R13.

6. de Lange T. Shelterin: the protein complex that shapes and safeguards human telomeres. Genes Dev. 2005; 19(18):2100-10.

7. Bilaud T, Brun C, Ancelin K, Koering CE, Laroche T and Gilson E. Telomeric localization of TRF2, a novel human telobox protein. Nat Genet. 1997; 17(2):236-9. 
8. Broccoli D, Smogorzewska A, Chong L and de Lange T. Human telomeres contain two distinct Myb-related proteins, TRF1 and TRF2. Nat Genet. 1997; 17(2):231-5.

9. Chong L, van Steensel B, Broccoli D, Erdjument-Bromage H, Hanish J, Tempst P and de Lange T. A human telomeric protein. Science. 1995; 270(5242):1663-7.

10. Celli GB and de Lange T. DNA processing is not required for ATM-mediated telomere damage response after TRF2 deletion. Nat Cell Biol. 2005; 7(7):712-8.

11. Richter T, Saretzki G, Nelson G, Melcher M, Olijslagers $\mathrm{S}$ and von Zglinicki T. TRF2 overexpression diminishes repair of telomeric single-strand breaks and accelerates telomere shortening in human fibroblasts. Mech Ageing Dev. 2007; 128(4):340-5.

12. Smith S, Giriat I, Schmitt A and de Lange T. Tankyrase, a poly(ADP-ribose) polymerase at human telomeres. Science. 1998; 282(5393):1484-7.

13. Smith $\mathrm{S}$ and de Lange T. Tankyrase promotes telomere elongation in human cells. Curr Biol. 2000; 10(20):1299302.

14. Chang W, Dynek JN and Smith S. TRF1 is degraded by ubiquitin-mediated proteolysis after release from telomeres. Genes Dev. 2003; 17(11):1328-33.

15. Cook BD, Dynek JN, Chang W, Shostak G and Smith S. Role for the related poly(ADP-Ribose) polymerases tankyrase 1 and 2 at human telomeres. Mol Cell Biol. 2002; 22(1):332-42.

16. Seimiya $\mathrm{H}$ and Smith $\mathrm{S}$. The telomeric poly(ADP-ribose) polymerase, tankyrase 1 , contains multiple binding sites for telomeric repeat binding factor 1 (TRF1) and a novel acceptor, 182-kDa tankyrase-binding protein (TAB182). J Biol Chem. 2002; 277(16):14116-26.

17. Dregalla RC, Zhou J, Idate RR, Battaglia CL, Liber HL and Bailey SM. Regulatory roles of tankyrase 1 at telomeres and in DNA repair: suppression of T-SCE and stabilization of DNA-PKcs. Aging (Albany NY). 2010; 2(10):691-708.

18. Huang SM, Mishina YM, Liu S, Cheung A, Stegmeier F, Michaud GA, Charlat O, Wiellette E, Zhang Y, Wiessner S, Hild M, Shi X, Wilson CJ, Mickanin C, Myer V, Fazal A, et al. Tankyrase inhibition stabilizes axin and antagonizes Wnt signalling. Nature. 2009; 461(7264):614-20.

19. Eller MS, Li GZ, Firoozabadi R, Puri N and Gilchrest BA. Induction of a p95/Nbs1-mediated $\mathrm{S}$ phase checkpoint by telomere 3' overhang specific DNA. FASEB J. 2003; 17(2):152-62.

20. Li GZ, Eller MS, Firoozabadi R and Gilchrest BA. Evidence that exposure of the telomere 3' overhang sequence induces senescence. Proc Natl Acad Sci U S A. 2003; 100(2):52731.

21. Li GZ, Eller MS, Hanna K and Gilchrest BA. Signaling pathway requirements for induction of senescence by telomere homolog oligonucleotides. Exp Cell Res. 2004; 301(2):189-200.

22. Rankin AM, Sarkar S and Faller DV. Mechanism of
T-oligo-induced cell cycle arrest in Mia-PaCa pancreatic cancer cells. J Cell Physiol. 2012; 227(6):2586-94.

23. Longe HO, Romesser PB, Rankin AM, Faller DV, Eller MS, Gilchrest BA and Denis GV. Telomere homolog oligonucleotides induce apoptosis in malignant but not in normal lymphoid cells: mechanism and therapeutic potential. Int J Cancer. 2009; 124(2):473-82.

24. Eller MS, Liao X, Liu S, Hanna K, Backvall H, Opresko PL, Bohr VA and Gilchrest BA. A role for WRN in telomerebased DNA damage responses. Proc Natl Acad Sci U S A. 2006; 103(41):15073-8.

25. Levy C, Khaled M and Fisher DE. MITF: master regulator of melanocyte development and melanoma oncogene. Trends Mol Med. 2006; 12(9):406-14.

26. Polager S and Ginsberg D. p53 and E2f: partners in life and death. Nat Rev Cancer. 2009; 9(10):738-48.

27. He G, Kuang J, Huang Z, Koomen J, Kobayashi R, Khokhar AR and Siddik ZH. Upregulation of p27 and its inhibition of CDK2/cyclin E activity following DNA damage by a novel platinum agent are dependent on the expression of p21. Br J Cancer. 2006; 95(11):1514-24.

28. Gnanasekar M, Thirugnanam S, Zheng G, Chen A and Ramaswamy K. T-oligo induces apoptosis in advanced prostate cancer cells. Oligonucleotides. 2009; 19(3):287-92.

29. Ohashi N, Yaar M, Eller MS, Truzzi F and Gilchrest BA. Features that determine telomere homolog oligonucleotideinduced therapeutic DNA damage-like responses in cancer cells. J Cell Physiol. 2007; 210(3):582-95.

30. Sarkar S and Faller DV. T-oligos inhibit growth and induce apoptosis in human ovarian cancer cells. Oligonucleotides. 2011; 21(1):47-53.

31. Andersen MH, Svane IM, Becker JC and Straten PT. The universal character of the tumor-associated antigen survivin. Clin Cancer Res. 2007; 13(20):5991-4.

32. Grossman D, McNiff JM, Li F and Altieri DC. Expression and targeting of the apoptosis inhibitor, survivin, in human melanoma. J Invest Dermatol. 1999; 113(6):1076-81.

33. Trefzer U, Hofmann M, Reinke S, Guo YJ, Audring H, Spagnoli G and Sterry W. Concordant loss of melanoma differentiation antigens in synchronous and asynchronous melanoma metastases: implications for immunotherapy. Melanoma Res. 2006; 16(2):137-45.

34. Srinivasan R and Wolchok JD. Tumor antigens for cancer immunotherapy: therapeutic potential of xenogeneic DNA vaccines. J Transl Med. 2004; 2(1):12.

35. Eller MS, Yaar M and Gilchrest BA. DNA damage and melanogenesis. Nature. 1994; 372(6505):413-4.

36. Hadshiew IM, Eller MS, Gasparro FP and Gilchrest BA. Stimulation of melanogenesis by DNA oligonucleotides: effect of size, sequence and 5' phosphorylation. J Dermatol Sci. 2001; 25(2):127-38.

37. Pedeux R, Al-Irani N, Marteau C, Pellicier F, Branche R, Ozturk M, Franchi J and Dore JF. Thymidine dinucleotides induce $\mathrm{S}$ phase cell cycle arrest in addition to increased 
melanogenesis in human melanocytes. J Invest Dermatol. 1998; 111(3):472-7.

38. Seimiya H. The telomeric PARP, tankyrases, as targets for cancer therapy. Br J Cancer. 2006; 94(3):341-5.

39. Li G, Tang L, Zhou X, Tron V and Ho V. Chemotherapyinduced apoptosis in melanoma cells is p53 dependent. Melanoma Res. 1998; 8(1):17-23.

40. Karlseder J, Broccoli D, Dai Y, Hardy S and de Lange T. p53- and ATM-dependent apoptosis induced by telomeres lacking TRF2. Science. 1999; 283(5406):1321-5.

41. Rankin AM, Faller DV and Spanjaard RA. Telomerase inhibitors and 'T-oligo' as cancer therapeutics: contrasting molecular mechanisms of cytotoxicity. Anticancer Drugs. 2008; 19(4):329-38.

42. Saretzki G, Sitte N, Merkel U, Wurm RE and von Zglinicki T. Telomere shortening triggers a p53-dependent cell cycle arrest via accumulation of G-rich single stranded DNA fragments. Oncogene. 1999; 18(37):5148-58.

43. Seimiya $H$, Muramatsu $Y$, Ohishi $T$ and Tsuruo $T$. Tankyrase 1 as a target for telomere-directed molecular cancer therapeutics. Cancer Cell. 2005; 7(1):25-37.

44. Riffell JL, Lord CJ and Ashworth A. Tankyrase-targeted therapeutics: expanding opportunities in the PARP family. Nat Rev Drug Discov. 2012; 11(12):923-36.

45. Byers HR, Etoh T, Doherty JR, Sober AJ and Mihm MC, Jr. Cell migration and actin organization in cultured human primary, recurrent cutaneous and metastatic melanoma. Time-lapse and image analysis. Am J Pathol. 1991; 139(2):423-35.

46. Puri N, Ahmed S, Janamanchi V, Tretiakova M, Zumba $\mathrm{O}$, Krausz T, Jagadeeswaran R and Salgia R. c-Met is a potentially new therapeutic target for treatment of human melanoma. Clin Cancer Res. 2007; 13(7):2246-53. 